\title{
ON RESIDUAL PROPERTIES
}

\author{
ALAN H. MEKLER ${ }^{1}$
}

\begin{abstract}
ABSTRACr. It is shown that the class of structures residually in a universal class closed under elementary equivalence is definable by a sentence of $L_{\infty}$.
\end{abstract}

In [2] and [3], Neumann showed the class of residually finite groups is of countable character; that is, a group is residually finite if every countable subgroup is. Sabbagh [4] gave a simple proof using ultraproducts of a generalization of this result. Here we use the completeness theorem to obtain a further generalization of this result. In addition we prove a definability theorem which implies the class of residually finite groups is definable by a sentence of $L_{\omega_{1} \omega}$. This answers a question raised in [4].

In the following we associate a finitary language with a class of structures in the normal manner and assume that similarity types match where necessary. We begin with some definitions. Suppose $X$ is a class of structures. A structure $A$ is residually in $X$ (denoted $A \in R X$ ) if for any two elements $a, b \in A$ with $a \neq b$ there is $C \in X$ and an onto homomorphism $f: A \rightarrow C$ such that $f(a) \neq f(b)$. A class of structures, $X$, is universal if each substructure of $X$ is itself an element of $X$. (Note $X$ is not necessarily an elementary class.) A theory $T$ is universal if the class of models of $T$ is universal. It is possible (cf. [1, Theorem 3.2.2, p. 124]) to give a syntactic characterization of universal theories. If $A$ is a structure, $D^{+}(A)$ will denote the set of atomic sentences, in the language with constants added for elements of $A$, true in $A$. (If $A$ is a group, $D^{+}(A)$ is the multiplication table.)

The following is an immediate consequence of the completeness theorem.

Lemma. Suppose $X$ is the class of models of a universal theory $T$, and $a, b \in A$. There exists an onto homomorphism $f: A \rightarrow C \in X$ with $f(a) \neq f(b)$ if and only if $T \cup D^{+}(A) \cup\{\neg(a=b)\}$ is consistent.

If $T$ is a set of sentences let $\Sigma(T)=\left\{\varphi\left(x_{0}, x_{1}, y_{0}, \ldots, y_{t}\right)\right\}$ $\varphi\left(x_{0}, x_{1}, y_{0}, \ldots, y_{t}\right) \rightarrow \neg \psi$ for some $\psi \in T$ and $\varphi\left(x_{0}, x_{1}, y_{0}, \ldots, y_{t}\right)$ is the conjunction of atomic formulae and $\neg\left(x_{0}=x_{1}\right)$ \}. From the lemma we have:

THEOREM 1. If $X$ and $T$ are as in the Lemma, the class $R X$ is axiomatized by

$$
\left\{\forall x_{0}, x_{1}, y_{0}, \ldots, y_{t} \neg \varphi\left(x_{0}, x_{1}, y_{0}, \ldots, y_{t}\right) \mid \varphi\left(x_{0}, x_{1}, y_{0}, \ldots, y_{t}\right) \in \Sigma(T)\right\} \text {. }
$$

We have, as well, the promised definability theorem.

Received by the editors April 21, 1978 and, in revised form, February 2, 1979.

AMS (MOS) subject classifications (1970). Primary 08A29, 08A25.

${ }^{1}$ Research supported by a grant from the National Research Council of Canada (Grant \# 3775). 
THEOREM 2. If $X$ is universal and closed under elementary equivalence, then the class $R X$ can be axiomatized by a sentence in $L_{\infty \omega}$.

Proof. By the hypothesis on $X$ we can choose $X_{i}, T_{i}(i \in I$, an index set) as in the Lemma such that $X=\bigcup_{i \in I} X_{i}$. The class $R X$ is axiomatized by:

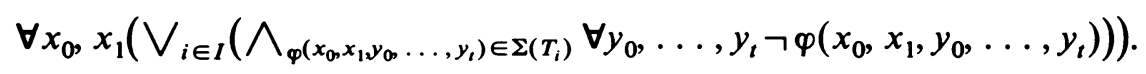

A structure $A$ satisfies the above sentence if and only if for any $a, b \in A$ with $a \neq b$ there is some $T_{i}$ such that $T_{i} \cup D^{+}(A) \cup\{\neg(a=b)\}$ is consistent.

By calculating the length of the above conjunctions and disjunction we have:

COROLLARY. The class of residually finite groups is axiomatizable by a sentence of $L_{\omega, \omega}$

Using the Löwenheim-Skolem theorem for $L_{\omega_{1} \omega}$ we can immediately conclude the class of residually finite groups is of countable character. In general we do not use Theorem 2 to show a structure is in $R X$.

THEOREM 3. Suppose $X$ is as in Theorem 2 and $\lambda$ is the cardinality of the language of $X$. If every substructure of $A$ of cardinality $\lambda$ is in $R X$ then $A \in R X$.

Proof. For $a, b \in A$ with $a \neq b$, choose $B \prec A$ ( $B$ an elementary substructure of $A$ ) such that $|B|=\lambda$ and $a, b \in B$. Since $B \in R X_{i}$ for some $i, B$ F $\left\{\forall y_{0}, \ldots, y_{t} \neg \varphi\left(a, b, y_{0}, \ldots, y_{t}\right) \mid \varphi\left(x_{0}, x_{1}, y_{0}, \ldots, y_{t}\right) \in \Sigma\left(T_{i}\right)\right\}$ (adopting the notation of Theorem 2). Since $B \prec A, T_{i} \cup D^{+}(A) \cup\{\neg(a=b)\}$ is consistent.

It is possible to extend these results. Suppose $T$ is a theory such that a homomorphic image of a model of $T$ is itself a model of $T$ (see [1, Theorem 3.2 .2$, p. 126] for a characterization of such theories). We can now relativize to $T$. Define $X$ to be universal relative to $T$ if a model of $T$ which is a substructure of some element of $X$ is itself an element of $X$. For such $X$ we can derive the above results for models of $T$ being residually in $X$. This discussion gives the generalization promised in the introduction.

The referee has pointed out a different generalization. The results hold for an $X$ not necessarily universal if $S P X$ is used in place of $R X$ ( $S$ denotes taking substructures and $P$ denotes taking Cartesian products).

\section{REFERENCES}

1. C. C. Chang and H. J. Keisler, Model theory, North-Holland, Amsterdam, 1973.

2. B. H. Neumann, Properties of countable character, Proc. Internat. Congr. Math. (Nice, 1970), vol. 1, Gauthier-Villars, Paris, 1971, pp. 293-296.

3. Group properties of countable character, Selected Questions of Algebra and Logic, Izdat. “Nauka" Sibirsk. Otdel., Novosibirsk, 1973, pp. 197-204. MR 48 \#6254.

4. G. Sabbagh, On properties of countable character, Bull. Austral. Math. Soc. 4 (1971), 183-192.

Department of Mathematics, Carleton University, Ottawa, Ontario, Canada

Current address: Department of Mathematics, University of Western Ontario, London, Ontario, Canada 